\title{
Stress polishing demonstrator for ELT M1 segments and industrialisation
}

\author{
Emmanuel Hugot a , Anaïs Bernard a, Marie Laslandes ${ }^{b}$, Johan Floriot a, \\ Thibault Dufour ${ }^{c}$, Denis Fappani c, Jean Marc Combes ${ }^{c}$, Marc Ferrari ${ }^{a^{*}}$ \\ ${ }^{a}$ Laboratoire d'Astrophysique de Marseille, 38 rue F. Joliot-Curie, F-13388 Marseille Cedex 13, France \\ bSpace Structures Laboratory, California Institute of Technology, Pasadena, Califomia 91125, USA \\ ' SESO, Pôle d'activités des Milles, BP 55000, 13792 Aix-en-Provence Cedex 3 France
}

\begin{abstract}
After two years of research and development under ESO support, LAM and Thales SESO present the results of their experiment for the fast and accurate polishing under stress of ELT 1.5 meter segments as well as the industrialisation approach for mass production. Based on stress polishing, this manufacturing method requires the conception of a warping harness able to generate extremely accurate bending of the optical surface of the segments during the polishing. The conception of the warping hamess is based on finite element analysis and allowed a fine tuning of each geometrical parameter of the system in order to fit an error budget of $25 \mathrm{~nm}$ RMS over $300 \mu \mathrm{m}$ of bending peak to valley. The optimisation approach uses the simulated influence functions to extract the system eigenmodes and characterise the performance. The same approach is used for the full characterisation of the system itself. The warping harness has been manufactured, integrated and assembled with the Zerodur 1.5 meter segment on the LAM 2.5meter POLARIS polishing facility. The experiment consists in a cross check of optical and mechanical measurements of the mirrors bending in order to develop a blind process, ie to bypass the optical measurement during the final industrial process. This article describes the optical and mechanical measurements, the influence functions and eigenmodes of the system and the full performance characterisation of the warping harness.
\end{abstract}

\section{INTRODUCTION}

Pioneered by the German astronomer Bernhard Schmidt since the 1930's ', Stress Polishing ${ }^{2}$ is a performing manufacturing method for smooth aspherical optics ${ }^{3}$, developed since several years for the production of high quality telescopes and instruments. Schmidt produced the entrance lens of its wide field telescope using this method, by applying a pressure under a thin menis cus during spherical polishing, the warping of the substrate corresponding to the exact inverse of the final aspherical shape. Improved by Lemaitre ${ }^{4}$ in 1974 , this production method allowed the polishing of almost 100 Schmidt plates between 1975 and 1990.

Stress polishing was then applied in the 1980's for the manufacturing of the twin Keck telescopes primary mirrors ${ }^{5,6}$. Nelson and Lubliner proposed a technological breakthrough allowing the increase of telescopes aperture by using the segmentation of primary mirrors. With a diameter of $10 \mathrm{~m}$, these telescopes are based on this technology. Nelson et al proposed the analytical expansion of conic surfaces, which defines an off axis section of a parabola (OAP) as a spherical surface plus some low order aberrations (Astigmatism and Coma mainly). A specific stress polishing method has been developed for the production of the Keck primary mirrors segments. However, due to the lack of simu lation tools, some second order effects were not anticipated and led to residual local errors on the segments, then removed by ion beam finishing.

This method is now under study to enable the development of future Extremely Large Telescopes (ELTs). This class of giant telescopes which diameter ranges from $25 \mathrm{~m}$ up to $39 \mathrm{~m}$ require technological breakthrough to see their first light. Three ELT projects can be highlighted: the Giant Magellan Telescope (GMT), 25m in diameter, made of 7 circular segments $8.4 \mathrm{~m}$ each, The Thirty Meter Telescope (TMT), 30m in diameter, made of almost 500 hexagonal segments $1.5 \mathrm{~m}$ each, and the European Extremely Large Telescope led by the European Southern Observatory (ESO), $39 \mathrm{~m}$ in diameter, which M1 will be made of almost 800 off axis segments of parabola, $1.5 \mathrm{~m}$ corner to comer. In such a context, the optimization of the manufacturing process of hexagonal OAP is a real challenge, and is the aim of the study presented hereafter.

*Principal Investigator 


\section{THE E-ELT PRIMARY MIRROR}

\subsection{Description of the E-ELT M1}

Despite the fact that the actual E-ELT baseline considers a $39 \mathrm{~m}$ primary mirror, the study presented in this article started in the frame of the $42 \mathrm{~m}$ E-ELT design, meaning that all the calculations corresponds to this study case. The primary mirror is a fast F/1 mirror, $42 \mathrm{~m}$ in diameter, with a Radius of curvature (Roc) of $84 \mathrm{~m}$, a conic constant of -0.993295 , and a central hole of diameter $12.720 \mathrm{~m}$. The primary is made of 984 hexagonal off axis segments. According to ESO description of E-ELT M1 geometrical properties ${ }^{6}$, the study case is applied to segment S3.16.16, with an off axis distance of $19.68 \mathrm{~m}$.

\subsection{Goal of the LAM/Thales SESO study}

The study performed at LAM is the unique academic study for the manufacturing of ELT segments. However, the aim of the study is to propose and demonstrate this technique by identifying the key points and propose technical solutions to be transferred to industrial partners.

LAM proposed a demonstration of the stress polishing method on a full scale prototype. To reach this goal, some key points have been identified and must be validated. The main goals of the experiment are the following:

- Design, manufacture, assemble and equip a complete warping harness dedicated to the E-ELT M1 segments mass production

- Characterise the equipped warping tool to extract the gain, offset, linearity and hysteresis of the actuators,

- Measure the influence functions of the system with an optical test set-up, a set of mechanical gauges and a set of force gauges.

- Estimate the performance of the warping tool with these results,

- Cross check the results and extract the more relevant measuring system to reach a goal of $1 \mu \mathrm{m}$ RMS WFE

- Characterise the repeatability of the warping, the stability in time and under polishing conditions

- Transfer the results to the industrial partner

Considering these functions, some specific devices have been chosen on the harness design, based on results given by finite elements analysis that are presented in the next sections.

\section{E-ELT SEGMENT OFF AXIS FORMULATION}

The following aims at defining clear links between the optical modes describing the shape of the segments and the mechanical deformations to be applied during the stress polishing. After a brief review of formulas giving the asphericities and Zernike coefficients to be generated, calculations are applied on the Segment S3 16.16

\subsection{Off axis Parabolas formulation}

An off axis parabola (OAP) can be defined with the following parameters: the F-ratio of the parent parabola, the F-ratio of the OAP, the off axis distance R. The general formulation giving the sag of a conic surface is given by:

$$
z_{c}(r)=\frac{r^{2}}{k+\sqrt{k^{2}-(K+1) r^{2}}}
$$

Where $\boldsymbol{r}$ is the circular coordinate, $\boldsymbol{k}$ is the radius of curvature and $K$ the conic constant. The general formulation of a sphere of radius $\boldsymbol{L}$ is given by:

$$
z_{s}(r)=L-\sqrt{L^{2}-r^{2}}
$$


Our interest is to express an off-axis section of a conic in local coordinates, in order to define its surface sag. Let us consider a circular section of the parabola, with $\boldsymbol{a}$ the radius of the circular section, $\boldsymbol{R}$ the off-axis distance of the section, $\rho=r / a$ the normalised radius, $\varepsilon=R / k$ the reduced parameter.

The conic surface sag in local radial coordinates is expresses by a sum of monomial terms

$$
z(\rho, \theta)=\alpha_{20} \rho^{2}+\alpha_{22} \rho^{2} \cos (2 \theta)+\alpha_{31} \rho^{3} \cos (\theta)+\alpha_{33} \rho^{3} \cos (3 \theta)+\alpha_{40} \rho^{4}+\alpha_{42} \rho^{4} \cos (2 \theta)
$$

where the $\alpha_{i j}$ coefficients are currently called asphericity coefficients, and depend on the dimensions of the off-axis section. They are expressed with the following formulas:

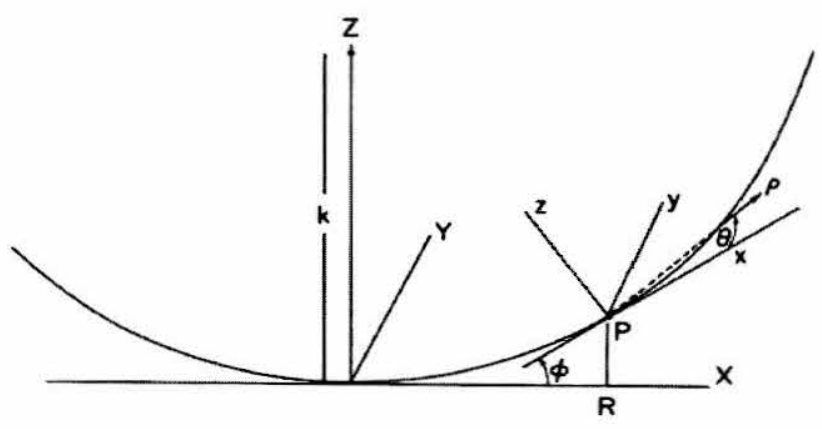

$$
\left\{\begin{array}{l}
\alpha_{20}=\frac{a^{2}}{k}\left\lfloor\frac{2-\Lambda \epsilon^{-}}{4\left(1-K \epsilon^{2}\right)^{\frac{3}{2}}}\right] \\
\alpha_{22}=\frac{a^{2}}{k}\left[\frac{K \epsilon^{2}}{4\left(1-K \epsilon^{2}\right)^{\frac{3}{2}}}\right] \\
\alpha_{31}=\frac{a^{3}}{k^{2}}\left[\frac{K \epsilon\left[1-(K+1) \epsilon^{2}\right]^{\frac{1}{2}}\left(4-K \epsilon^{2}\right)}{8\left(1-K \epsilon^{2}\right)^{3}}\right] \\
\alpha_{33}=\frac{a^{3}}{k^{2}}\left[\frac{K^{2} \epsilon^{3}\left[1-(K+1) \epsilon^{2}\right]^{\frac{1}{2}}}{8\left(1-K \epsilon^{2}\right)^{3}}\right] \\
\alpha_{40}=\frac{a^{4}}{k^{3}}\left[\frac{8(K+1)-24 K \epsilon^{2}+3 K^{2} \epsilon^{4}(1-3 K)-K^{3} \epsilon^{6}(2-K)}{64\left(1-K \epsilon^{2}\right)^{\frac{9}{2}}}\right] \\
\alpha_{42}=\frac{a^{4}}{k^{3}}\left[-\frac{K^{2} \epsilon^{2}\left(1+5 K-K \epsilon^{2}(6+5 K)\right)}{16\left(1-K \epsilon^{2}\right)^{\frac{7}{2}}}\right]
\end{array}\right.
$$

One of the main interests of the stress polishing technique is the possibility of using the same polishing sphere radius from one segment to the others. That allows minimising the deformations to be applied on the substrate and also better guaranty the continuity of curvature between segments. The radius of this sphere denoted $\boldsymbol{L}$ is the best fit sphere radius of the average Focus asphericity $\alpha_{20 a v e}$ over the whole primary mirror aperture. These terms are given by:

$$
L=\frac{2 k\left(1-K \frac{\epsilon_{\max }^{2}}{2}\right)^{\frac{3}{2}}}{2-K \frac{\epsilon_{\max }^{2}}{2}} \quad \alpha_{20 a v e}=\frac{a^{2}}{k} \frac{2-K \frac{\epsilon_{\max }^{2}}{2}}{4\left(1-K \frac{\epsilon_{\max }^{2}}{2}\right)^{\frac{3}{2}}}
$$

It is useful to express the asphericities in terms of Zernike polynomials. Denoting $Z_{i j}$ the Zemike coefficient of radial order $\boldsymbol{i}$ and angular order $\boldsymbol{j}$, and $\boldsymbol{C}_{i j}$ the associated coefficient, we use the following relations:

$$
\begin{array}{rlr}
C_{20} & =\frac{\alpha_{20}}{2}+\frac{\alpha_{40}}{2}+\ldots & (\text { Focus 1) } \\
C_{22}=\alpha_{22}+\frac{3}{4} \alpha_{42}+\ldots & (\text { Astigmatism 3) } \\
C_{31}=\frac{\alpha_{31}}{3}+\ldots & (\text { Coma 3) } \\
C_{33}=\alpha_{33}+\ldots & (\text { Trefoil 5) } \\
C_{40}=\frac{\alpha_{40}}{6}+\ldots & (\text { Spherical 3) } \\
C_{42}=\frac{\alpha_{42}}{4}+\ldots & (\text { Astigmatism 5) }
\end{array}
$$

Applying these equations to segment S3.16.16 allows calculating the best fit sphere radius and the values of aberrations to be generated on the optical surface (Table 1). 
Table 1 Asphericities, Zemike coefficients and RMS Zernike coefficients to be generated on the S3.16.16 segment

\begin{tabular}{|cr|cr|cr|}
\hline \multicolumn{2}{|c|}{ Segment surface asphericity $[\boldsymbol{\mu m}]$} & \multicolumn{2}{|c|}{ Zernike Coefficients $[\boldsymbol{\mu m}]$} & RMS Zernike Coefficients $[\boldsymbol{\mu m}]$ \\
\hline Focus 1 & +77.603 & Focus 1 & +38.801 & Focus 1 & $+\mathbf{2 2 . 4 0 2}$ \\
Astm 3 & +76.071 & Astm3 & +76.071 & Astm3 & $+\mathbf{+ 3 1 . 0 5 6}$ \\
Coma 3 & +5.155 & Coma 3 & +1.719 & Coma 3 & $+\mathbf{+ 0 . 6 0 8}$ \\
Trefoil 5 & -0.070 & Trefoil 5 & -0.070 & Trefoil 5 & $\mathbf{- 0 . 0 2 5}$ \\
Sphe 3 & -0.007 & Sphe 3 & -0.001 & Sphe 3 & $<\mathbf{0 . 0 0 1}$ \\
Astm 5 & -0.005 & Astm 5 & -0.001 & Astm 5 & $<\mathbf{0 . 0 0 1}$ \\
\hline
\end{tabular}

\section{STRESS POLISHING OF E-ELT SEGMENT PROTOTYPE}

In order to warp the mirror into the desired shape, some specific parameters of the warping harness have been optimized using finite element analysis. Results are presented hereafter, with a focus on the interface between harness and mirror. The harness will be glued on the circular blank, and this interface has been optimized in order to minimize the stress within the blank and the glue, and to minimize the warping residuals in terms of optical quality.

\subsection{Mechanical design of the warping harness}

Based on Active Optics concepts developed by Lemaitre et al ${ }^{2}$, a circular blank can be bent into optical modes by using the correspondence between mechanical Clebsch-Jordan modes and Zernike modes. The design presented on Figure 1 uses bending arms clamped at the edge of a thick circular ring, acting as a local stress filter; forces are applied as far as possible from the optical surface, according to Saint Venant's principle ${ }^{7}$. Forces are applied at the end of each arm, allowing transferring net shearing forces and bending moments to the optical meniscus. The number of arms is quite high in order to have a good continuity of the deformation at blank edges and a smooth stress repartition. Harness material is stainless steel (AISI 316L). Its dimensions have been optimized regarding the following parameters:

- Warping performance in terms of residuals

- Displacements and forces needed to generate the deformation

- Resulting stress in Zerodur

The glue is modelled as a uniform band of $150 \mu \mathrm{m}$ thickness. Properties of Scotch Weld DP460 are used (TBC). To minimize the stress in the material, the gluing area is extended with a conge of $15 \mathrm{~mm}$ radius between the hamess and the mirror.
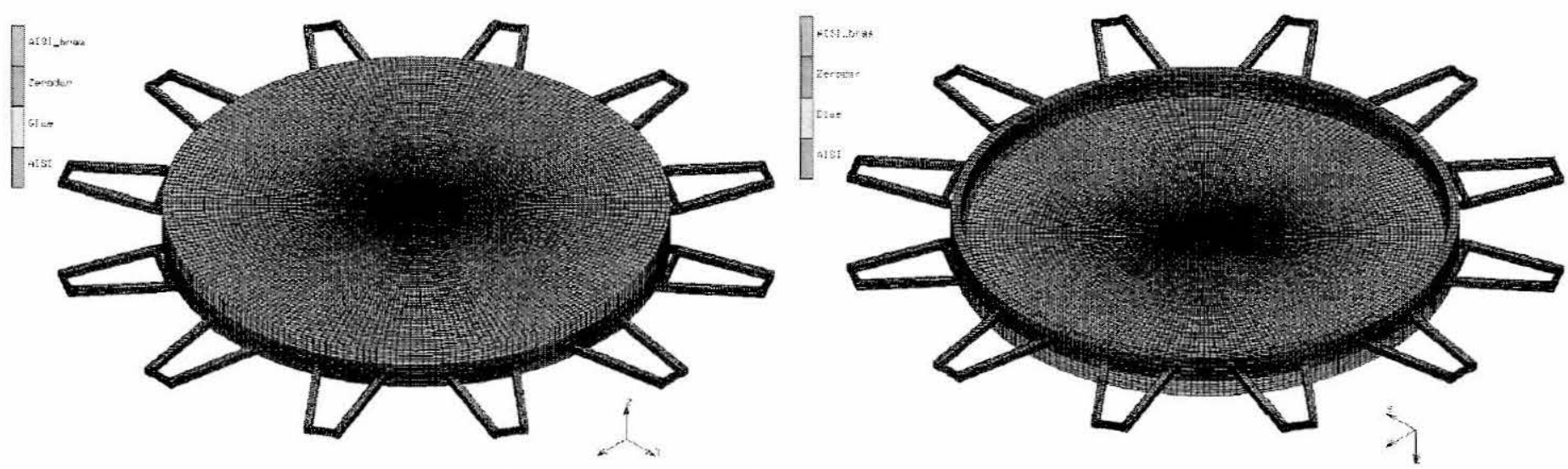

Figure 1 Finite Element Model of the substrate and the warping system(98976 elements - 127398 nodes). 


\subsection{Finite element analysis / Performances on full size}

\subsubsection{Boundary conditions}

Actuators can be simulated either by the application of a force or a displacement. These two approaches are similar and conduct to the same results. As explained before, our system has 24 degrees of freedom. Displacements (in the optical axis direction) are applied at the location of actuators. Note that if the actuators are driven in displacement; the resulting forces are computed in the FEA. A $25^{\text {th }}$ actuator is defined: it applies a uniform load on the mirror back face to compensate for the spherical aberration. To hold the system in the (xy) plan, isostatic initial conditions are defined.

\subsubsection{Analysis method}

Once the system is defined, its behaviour can be studied for the generation of the required deformation. The analys is is based on the system Influence Functions (IF). One Influence Function is the phase map resulting from the unit command on one actuator. The projection of the required mirror shape on the bas is containing the 25 system IF ( 24 actuators applying displacements and one applying pressure) gives the command for each actuator. This approach also allows calculating the system Eigen modes, as illustrated on Figure 3. Then, the system performances are deduced:

- The deformation generated by the system can be reconstructed and compared to the required deformation. From that, the residual phase map is deduced.

- As explained, projection coefficients gives the actuators needed performances (forces and displacements)

- By applying the right actuators commands on the finite elements model, stress in the system is estimated.

Figure 2 illustrates this last point. FEA has been performed on the full size model, $1,52 \mathrm{~m}$ in diameter, in order to accurately calculate the stress within the Zerodur blank and the metallic harness. More than 100,000 elements were used in the model to estimate the residual error on Mid-high spatial frequency up to 50 cycles per pupil. With an amount of stress about $4 \mathrm{MPa}$, the blank has a very low probability of breakage.

Figure 4 shows the system performance. The phase map corresponding to the deformation is projected on the Eigen modes basis, allowing its reconstruction with the system modes. The difference between the two maps gives the amount of residuals that is lower than $20 \mathrm{~nm}$ RMS, fully compliant with our goal of $1 \mu \mathrm{m}$ RMS. This very good result is extremely important as it allows relaxing the error budget allocated on other error sources such as polishing, testing, thermal control, etc...

However, additional simulations have been performed in order to estimate the amount of residual after the finishing process. Considering IBF, we choose a FWHM of $50 \mathrm{~mm}$ with an efficiency of $100 \%$. Results of simulations show that after only one run, the residual error is almost fully corrected, down to $3 \mathrm{~nm}$ RMS.
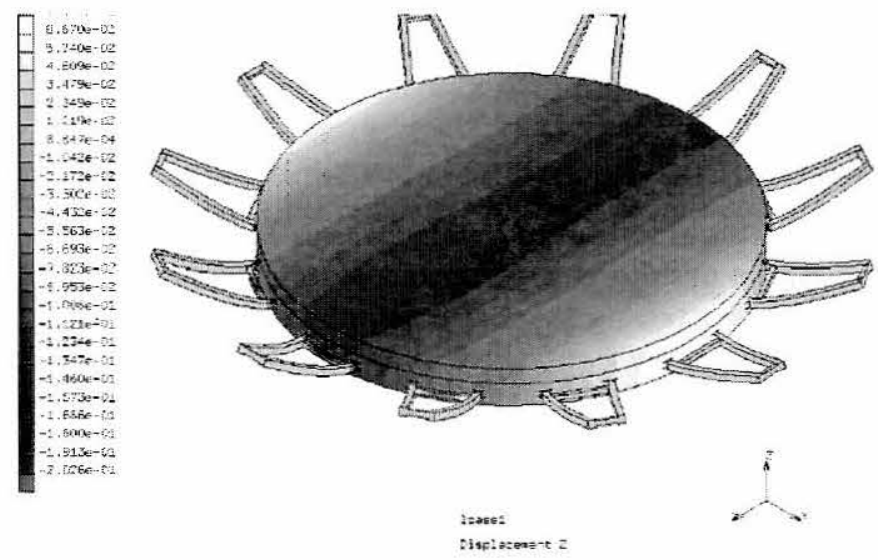

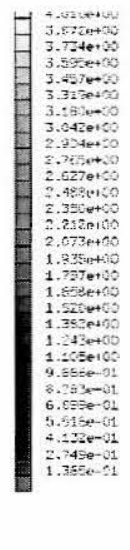

Figure 2 Finite element analysis of the $1.52 \mathrm{~m}$ warping harness. The deformation on the pupil $(1.425 \mathrm{~m})$ is equal to $250 \mu \mathrm{m}$ PV. Right: Repartition of equivalent Von Mises stress on the rear face of the ZERODUR blank. The amount of stress is about $4 \mathrm{MPa}$, quite far from the breakage limit of ZERODUR. 

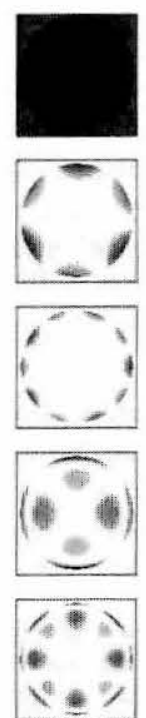
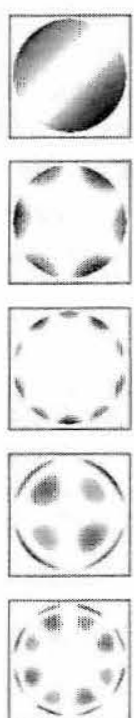
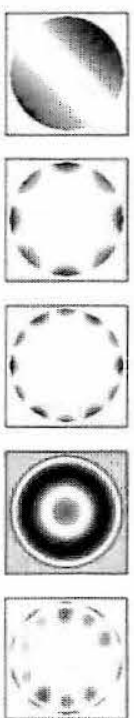
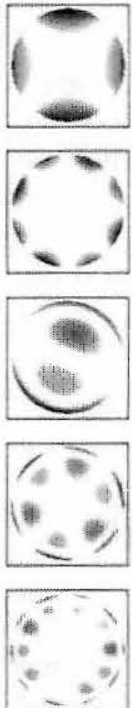
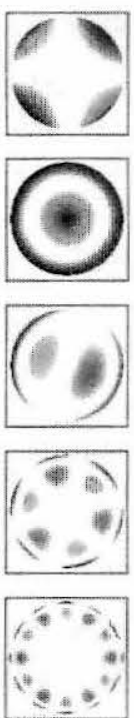

Figure 3 The influence function approach allows knowing the eigen modes of the system. The 24 actuators give 24 eigen modes, corresponding to the first low order optical aberrations.
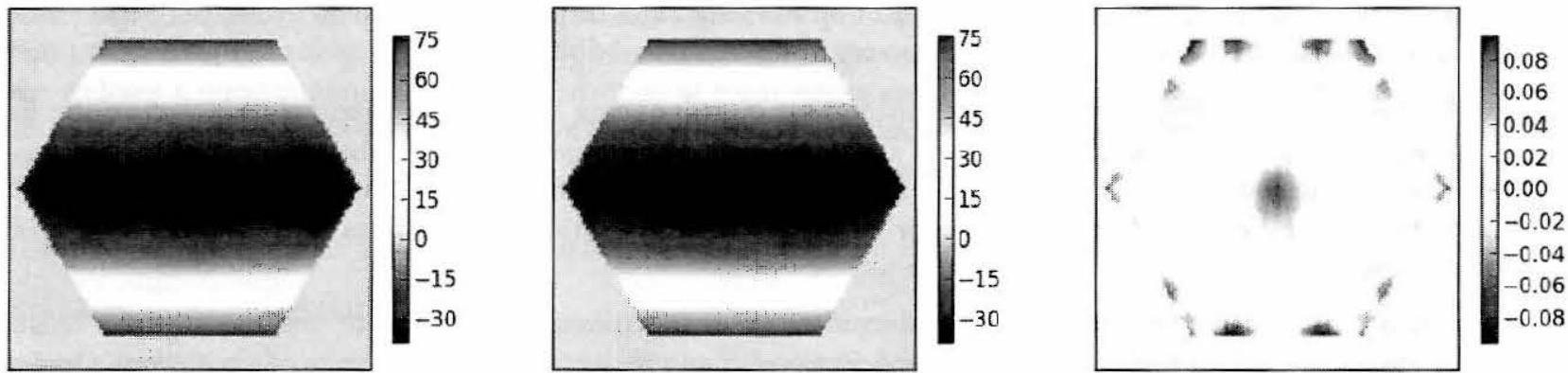

Figure 4 Performance of the warping system. The phase map to be reconstructed is projected on the eigen modes basis. Residuals of the reconstruction about 20nm RMS. Numerical simulations show that after the IBF finishing process, residuals are lower than $3 \mathrm{~nm}$ RMS.

\section{POLARIS 2.5M POLISHING FACILITY AND OPTICAL TEST BENCH}

LAM is equipped with a $2.5 \mathrm{~m}$ polishing machine, able to use either computer control polishing or classical polishing with full sized tools. This facility named POLARIS (POLishing Active and Robotic Integrated System), dedicated to research and development programs, is installed in LAM building under a testing tower in order to directly control the optical quality of manufactured pieces during polishing, allowing a gain in terms of operations count. On Figure 5 are shown the POLARIS machine and the integrated warping harness, interfaced with the polishing rotating plate. This very thick plate acts as an ultra-stable reference. 


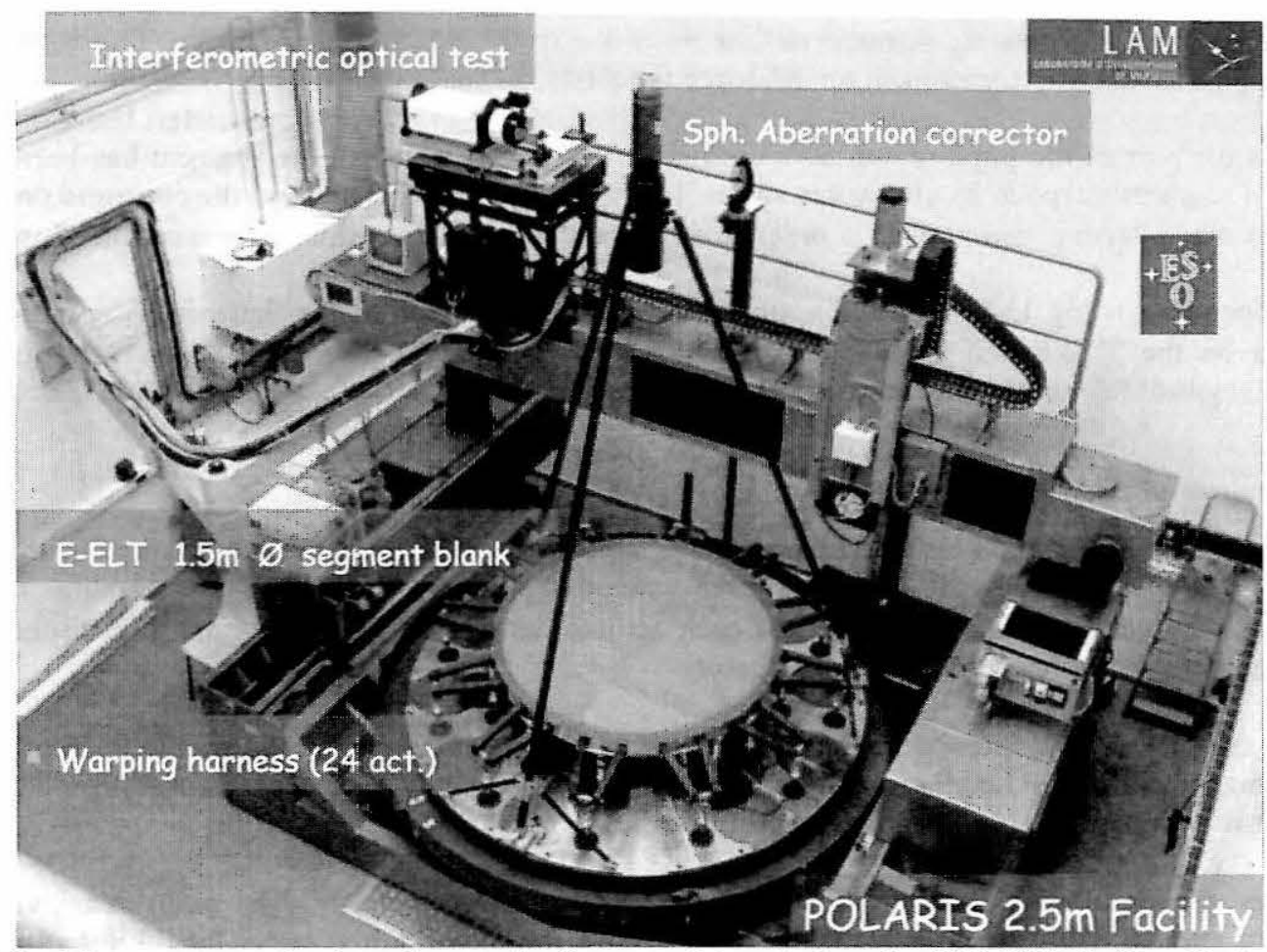

Figure 5 The $1.5 \mathrm{~m}$ segment, glued on the warping harness, integrated on the POLARIS facility. Optical tests are performed with interferometry and Shack Hartmann WFS.

\subsection{Test bench}

A specific design has been developed using a fast folding sphere (Diameter $1.5 \mathrm{~m}, \mathrm{~F} / 4$ ) placed at $9 \mathrm{~m}$ above the experiment on top of LAM testing tower. This configuration allows reducing the length of the test setup from $84 \mathrm{~m}$ down to $14 \mathrm{~m}$. As the main objective of the study is to demonstrate the optical quality of the warping, working in a differential configuration could be sufficient: a calibration of the test setup is necessary to fix the reference, but theoretically, aberrations of the setup will not limit the performance of the system. However, in order to work as close as possible to a zero-aberration mode, aberrations of the setup will be compensated using optical correctors placed close to the focus. The illuminating mirror has a focal plane at $6 \mathrm{~m}$, where flat folding mirror is placed to send the beam to the interferometer and to a $64 \times 64$ Shack Hartmann wave front sensor. Close to the focus, correcting lenses are placed in order to compensate for test bench aberrations.

Three configurations have been defined:

$\checkmark$ Testing the spherical mirror

$\checkmark$ Testing the warped mirror

$\checkmark$ Testing the polished mirror (inverse of configuration 2)

In addition, 25 LVDT devices are sensing the back face of the segment, and provide a low resolution mechanical measurement. The aim of the experiment will be to demonstrate that the command using LVDT data is enough sufficient to recover a quality within the range of the $1 \mu \mathrm{m}$ RMS specification.

\section{WARPING PERFORMANCE ESTIMATION}

\subsection{Segment flattening}

LVDT and SHWS measurements made before and after gluing showed that the glue reticulation had a non-negligible impact on the segment shape (typically $6 \mu \mathrm{m}$ RMS). This deformation has been monitored and will be included in the final deformation of the segment during polishing phase, with respect to its initial shape. 
SHWS measurements showed a strong distortion of the pupil due to this segment deformation. This implies that optical measurements cannot be used at this moment for influence functions calculations.

Using the accessible part of the pupil (about $80 \%$ of the total one), a flattening of the segment has been performed to recover the initial segment shape in its stress free shape. This fundamental step validated the command procedure based on LVDT data. A mode filtering was applied in order to minimise the commands while optimising the shape generation.

The flattening operation using LVDT IF was a success. As a consequence of the flattening, the optical pupil then becomes circular on the SHWS and the full characterisation of the system with optical measurements of influence functions on the segment full-aperture is now possible.

\subsection{Characterisation of actuators and system}

Each actuator has been characterized by measuring 10 times its optical influence function (measured by the SHWS). The force applied is $30 \mathrm{daN}$, i.e. typically $5 \%$ of the expected value to be applied for the segment warping. This procedure allows evaluating the average influence function of each actuator as well as the dispersion. The dispersion between actuator is also extracted to compute the gain per actuator.

Figure 6 shows the 12 averaged internal influence functions. The dispersion (calculated by the RMS of the difference between the averaged influence functions and a particular one) is typically less than $20 \mathrm{~nm}$ RMS which is perfectly sufficient for future operations.

Table 2 indicates the mean sensitivities $(\mathrm{nm} / \mathrm{daN})$ of the internal and external IF. The mean sensitivities are quite identical for both internal and external influence functions while the dispersion is larger for the external ones. These results are fundamental to extract the gain for each actuator.

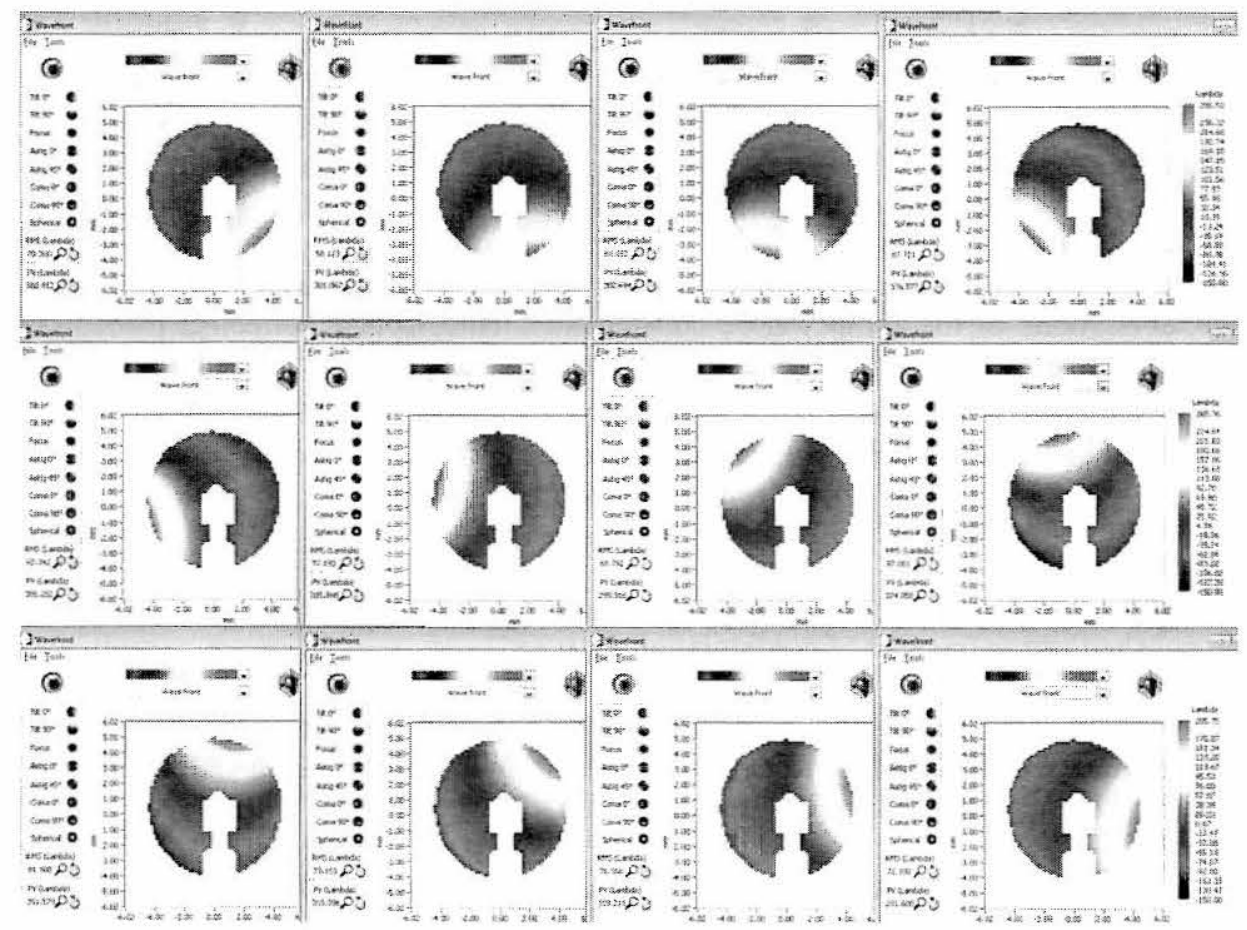

Figure 6 Averaged influence functions of internal actuators, measured with the $64 \times 64$ SHWS 
Table 2 Mean sensitivities $(\mathrm{nm} / \mathrm{daN})$ of the internal and external IF. The dispersion between actuators allows to calculate the gain to be applied on each of them to compute the command vector.

\section{Internal Actuator Sensitivity ( $\mathrm{nm} / \mathrm{daN})$ External Actuator Sensitivity (nm/daN)}

\begin{tabular}{c|ccc} 
A & 302.9 & B & 284.2 \\
\hline C & 301.2 & D & 390.9 \\
E & 302.7 & $\mathrm{~F}$ & 305.3 \\
G & 376.6 & $\mathrm{H}$ & 435.5 \\
J & 388.3 & $\mathrm{I}$ & 383.7 \\
K & 326.0 & $\mathrm{~L}$ & 278.4 \\
M & 299.9 & $\mathrm{~N}$ & 375.6 \\
$\mathrm{O}$ & 374.8 & $\mathrm{P}$ & 405.9 \\
$\mathrm{R}$ & 351.6 & $\mathrm{Q}$ & 277.2 \\
$\mathrm{~S}$ & 315.1 & $\mathrm{~T}$ & 321.4 \\
U & 319.2 & $\mathrm{~V}$ & 267.7 \\
W & 291.6 & $\mathrm{X}$ & 243.3 \\
& & & $\mathbf{3 3 0 . 8}$ \\
Mean & $\mathbf{3 2 9 . 2}$ & Mean & $\mathbf{6 1 . 4}$ \\
\hline Sigma & $\mathbf{3 3 . 0}$ & Sigma & \\
\hline
\end{tabular}

\subsection{Performance estimation}

Starting with the optical influence functions measurement, the eigenmodes of the system are computed. A strategy of modes filtering is applied in order to identify the best combination of modes allowing to reach the best optical quality while minimising the required energy to produce the segment off axis shape. Figure 7 draws the filtering results showing that the filtering of the last ten modes is the best trade off.
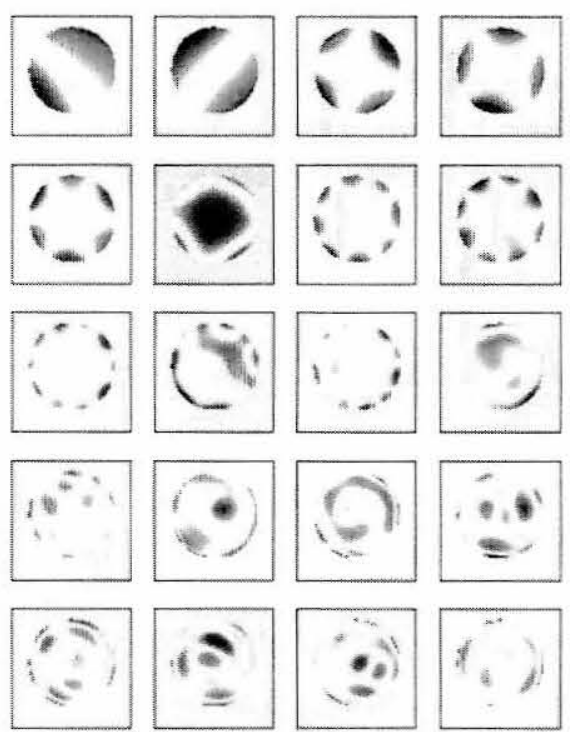

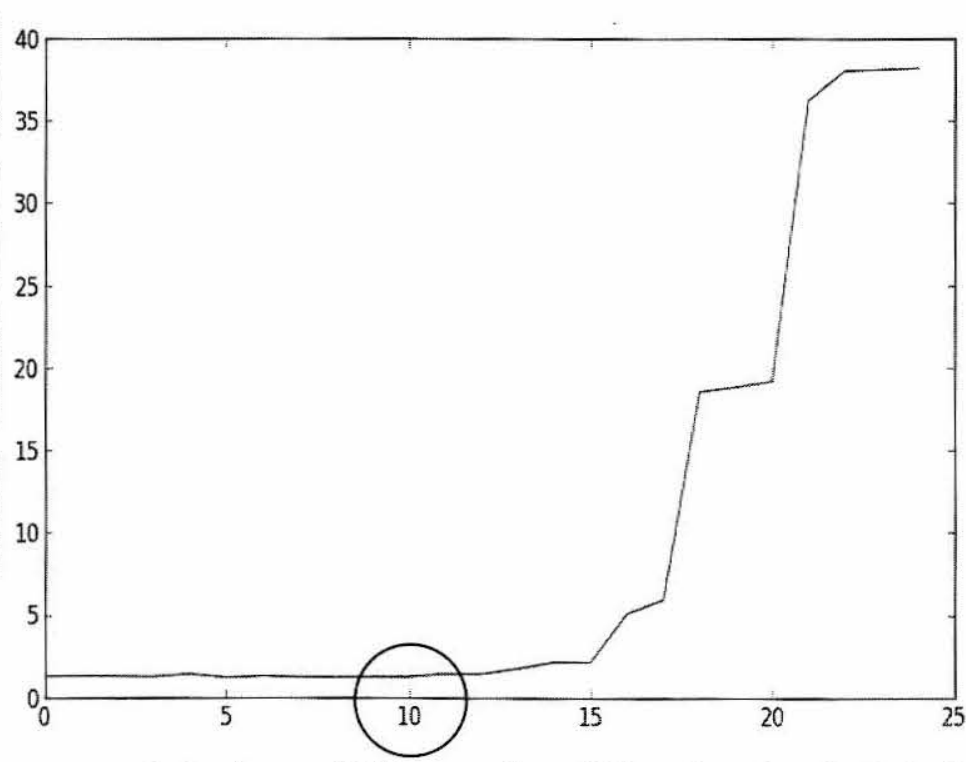

Figure 7 Eigen modes of the system and performance evolution in $\mu \mathrm{m}$ RMS vs number of filtered modes; Optimized number of filtered modes around 10 . 
Figure 8 present the very first results obtained from these measurements and optimizations. The system is able to provide the required shape with a precision of $1.15 \mu \mathrm{m}$ RMs after the first run. This extremely good preliminary result is very promising for the industrialization process. Experience taken from adaptive optics systems operations allows to anticipate a converging correction during the polishing phases that will reach $1 / 4^{\text {th }}$ of this estimated performance after successive iterations.

An industrialization approach will allow to reduce the number of iterations down to two, in order to optimize the polishing time. Such a process is clearly advantageous for the manufacturing of E-ELT segments, as it corresponds to the first phase of pre-polishing, before hexing and integration on the dedicated whiffle tree. The industrialization study is undertaken by Thales Seso, and will allow to propose an affordable process for these first stages of segment polishing.

The experiment is ongoing at LAM and Thales Seso. The next steps are the generation of the total shape, the repeatability and stability characterizations, as well as the implementation of a control strategy to avoid the optical testing during the final mass production of pre-polished segments. A particular emphasis on the high spatial frequency minimization is fundamental to provide high-quality polished circular segments, in order to minimize the number of runs during the finishing process.

Required shape
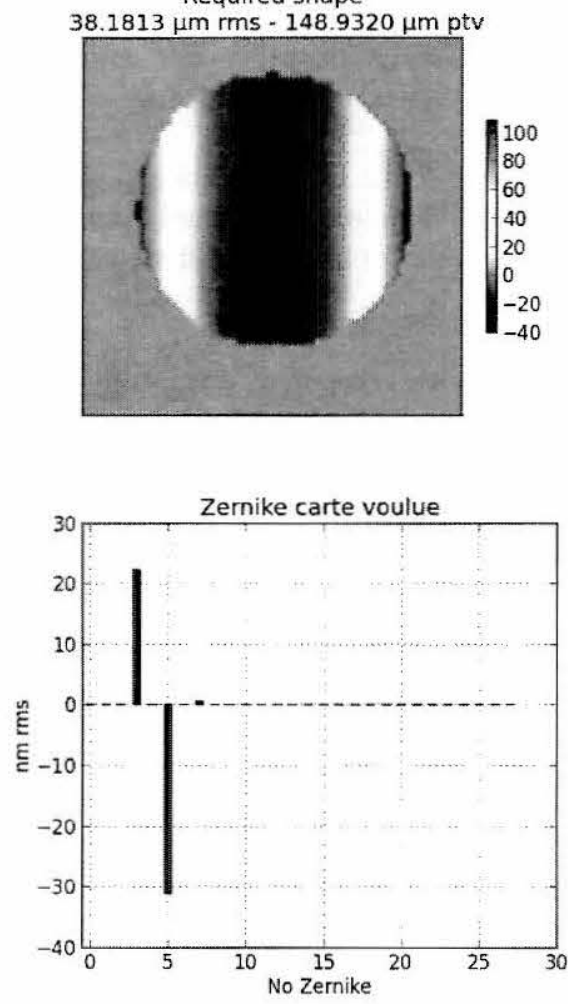

Generated shape
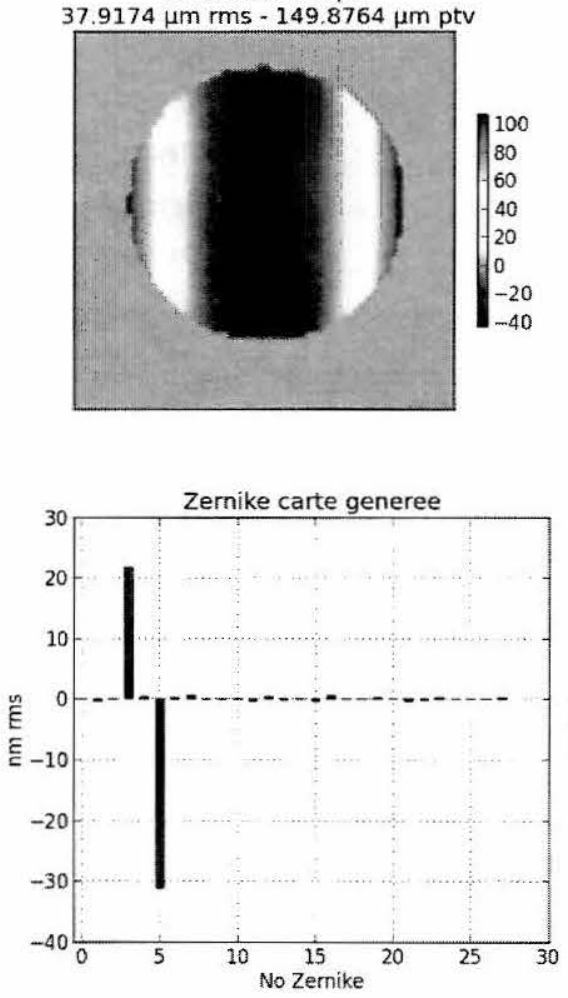

Residuals

$1.1571 \mu \mathrm{m}$ rms - $6.8587 \mu \mathrm{m}$ ptv
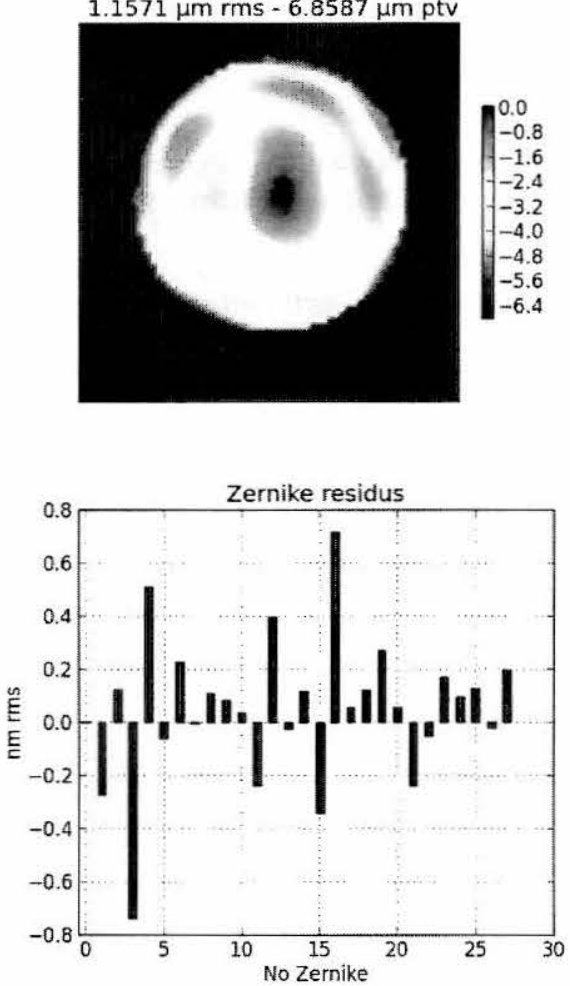

Figure 8 System performance estimation over the circular pupil, demonstrating that the first run could provide a shape which accuracy is of order of $1 \mu \mathrm{m}$ RMS. This preliminary result is extremely encouraging and confirms the precedent studies. A specific converging strategy will then be applied to decrease this value down to $250 \mathrm{~nm}$ RMS during the experiment. 


\section{CONCLUSION AND PERSPECTIVES}

Studies are ongoing at LAM for the demonstration of a full scale prototype of E-ELT segment, based on the stress polishing method. This fast-track study will produce two main results: $1 /$ the demonstration of the repeatability of the process, mandatory criterion for mass production of ELT segments, $2 /$ the production of a $1.5 \mathrm{~m}$ stress polished segment.

The harness design is made of a circular thick outer ring, glued on a circular Zerodur blank. Twelve arms are clamped on the ring to the transfer shearing forces and bending moments to the blank thanks to actuators placed at their extremities. The warping harness has been designed and optimized thanks to Finite Element Analysis. An approach based on the system characterization via influence functions and Eigen modes allows an accurate estimation of the expected residuals due to the warping hamess.

Simulation results show a final residual error of less than $20 \mathrm{~nm}$ RMS, validating the warping hamess design. This excellent result allows relaxing the specifications on other contributors in the error budget, such as integration, polishing or testing. Numerical simulations of the finishing process have shown that this error could be reduced to $3 \mathrm{~nm}$ RMS in one run, completely validating the mechanical design of the harness.

The full scale demonstrator is now integrated on the LAM $2.5 \mathrm{~m}$ polishing facility named POLARIS, placed inside a $10 \mathrm{~m}$ testing tower. A specific test setup has been designed, using a $1.5 \mathrm{~m}$ folding sphere and correctors placed closed to the focus. Three configurations have been optimized for the testing of the spherical mirror, the warped mirror and the final polished mirror.

Actions ongoing are the characterisation and optimisation of the system. Influence functions have been recorded with a $64^{2}$ Shack Hartmann WFS and Eigen modes have been extracted. The global performance of the system is es timated with these calculations and shows that the system is able to reach a performance of about $1 \mu \mathrm{m}$ RMS after the first run, which is an extremely promising result to go to a mass production process. The experiment also validates the command strategy based on LVDT sensing to avoid any optical testing during the production phases.

This demonstration will offer a unique technological breakthrough to the community, allowing proposing an affordable mass production process for the E-ELT segments.

\section{ACKNOWLEDGEMENTS}

This activity is conducted under ESO contract. The authors would like to thank J. Spyromilio and Marc Cayrel for their support on this project. The authors are also grateful to Fabrice Madec, Amandine Caillat and Mohamed Belhadi for their participation during integration and alignment.

\section{REFERENCES}

[1] Schmidt B., “A coma free telescope", Mitt. Hamb. Strenv. , 7, 15, 1932

[2] Lemaitre, G.R., "Astronomical optics and elasticity - Active Optics methods", (Berlin: Springer) 2009

[3] Hugot E. et al, "Active Optics methods for exoplanet direct imaging. Stress polishing of supersmooth aspherics for VLT-SPHERE planet finder", Astronomy \& Astrophysics 538 (2012)

[4] Lemaitre G.R., "New procedure for making Schmidt corrector plates ", Applied Optics, Vol.11, p.1630, 1972

[5] Lubliner J., Nelson J.E., "Stressed mirror polishing. 1: A technique for producing non axisymmetric mirrors", Applied Optics, vol.19, nr14, p.2332-2340, 1980

[6] Nelson J., Gabor G., Hunt L.K., Lubliner J., Mast T.S., "Stressed mirror polishing. 2: Fabrication of an off-axis section of a paraboloid", Applied Optics, vol.19, nr14, p.2341-2352, 1980

[7] St Venant B., Mémoires, tome 14, p65 